\title{
Three-Dimensional Primary Hepatocyte Culture in Synthetic Self-Assembling Peptide Hydrogel
}

\author{
SIHONG WANG, Ph.D., ${ }^{*}{ }^{\dagger}$ DEEPAK NAGRATH, Ph.D. ${ }^{\dagger}$ POHUN C. CHEN, B.S., \\ FRANÇOIS BERTHIAUME, Ph.D., and MARTIN L. YARMUSH, M.D., Ph.D.
}

\begin{abstract}
Drug metabolism studies and liver tissue engineering necessitate stable hepatocyte cultures that express liver functions for a minimum of 4 days to 3 weeks. Current techniques, using different biomaterials and geometries, that maintain hepatocellular function in vitro exhibit a low cell density and functional capacity per unit volume. Herein we investigated a well-defined synthetic peptide that can self-assemble into threedimensional interweaving nanofiber scaffolds to form a hydrogel, PuraMatrix, as a substrate for hepatocyte culture. Freshly isolated primary rat hepatocytes attached, migrated, and formed spheroids within 3 days after seeding on PuraMatrix. Hepatocytes expressed the apical membrane marker dipeptidyl peptidase IV at cell-cell contacts. Compared to the collagen sandwich, albumin and urea secretion on PuraMatrix were higher for the first week, and cytochrome P450IA1 activity was higher throughout the culture period. Mitochondrial membrane potential 1 day after seeding was higher on PuraMatrix than in the collagen sandwich, suggesting better preservation of the metabolic machinery. PuraMatrix and Matrigel showed similar albumin and urea production. PuraMatrix is an attractive system for generating hepatocyte spheroids that quickly restore liver functions after seeding. This system is also amenable to scale-up, which makes it suitable for in vitro toxicity, hepatocyte transplantation, and bioartificial liver development studies.
\end{abstract}

\section{INTRODUCTION}

$\mathbf{L}$ IVER TISSUE ENGINEERING has been under investigation for over 40 years to help recovery from acute liver diseases, provide a bridge to liver transplantation, and establish in vitro systems for drug metabolism and toxicity evaluation. Since primary hepatocytes lose their phenotype rapidly after isolation, maintaining liver-specific functions in vitro has been a major goal of these studies. ${ }^{1}$ Methods that can maintain stable long-term functions of hepatocytes use different biomaterials and culture configurations, including (1) the collagen sandwich configuration, ${ }^{2,3}$ and (2) three-dimensional spheroid culture induced by seeding on weakly adherent surfaces or soft gels, such as poly HEMA coatings, ${ }^{4,5}$ alginate sponges, ${ }^{6}$ and Matrigel $^{\mathrm{TM}},{ }^{7,8}$ or by preventing cell attachment by suspension culture in spinner flasks. ${ }^{9,10}$

Two-dimensional culture systems (e.g., collagen sandwich) can maintain the long-term hepatic phenotype, and are robust and easy to maintain ${ }^{11}$; however, they contain low cell numbers-and thus low functional capacity-per unit volume (typically $\sim 10^{6}$ cells $/ \mathrm{mL}$ ) and are difficult to adapt to a large-scale culture system. Three-dimensional culture methods are more amenable to scale-up and provide cells an opportunity to form a large number of intercellular contacts that help maintain their in vivo phenotype. ${ }^{12-14}$ Current methods to form hepatocyte spheroids yield aggregates with average diameters of $130 \mu \mathrm{m}$ in spinner flasks, ${ }^{10} 160 \mu \mathrm{m}$ on

Center for Engineering in Medicine, Massachusetts General Hospital, Shriners Burn Hospital, and Harvard Medical School, Boston, Massachusetts.

* Present address: Biomedical Engineering Department, The City College of New York, CUNY, New York, New York.

${ }^{\dagger}$ These authors contributed equally to this work. 
poly-HEMA coatings, ${ }^{4}$ and $100 \mu \mathrm{m}$ in alginate sponges. ${ }^{6}$ Spheroids greater than $100 \mu \mathrm{m}$ in diameter are subject to oxygen and nutrition delivery limitations to the center of the aggregates, which makes them unsuitable for long-term cultures. Smaller aggregates can be obtained on Matrigel, which is prepared from Engelbreth-Holm-Swarm sarcoma grown in mice. Although hepatocytes maintain a long-term function on Matrigel, the potential tumorigenicity and immunogenicity, as well as poorly defined composition and potential batch-tobatch variation, of this material limit its use for in vivo therapeutic applications and for in vitro drug toxicity studies.

In this study, we sought a well-defined synthetic hydrogel biomaterial that could promote cell attachment and migration to induce the formation of small hepatocyte spheroids. PuraMatrix $^{\mathrm{TM}}$ is a peptide-based hydrogel with over $99 \%$ water content that can self-assemble into three-dimensional interweaving nanofibers after adding a salt solution. ${ }^{15}$ The fiber itself is about $10 \mathrm{~nm}$, and the pore size ranges from 50 to $200 \mathrm{~nm}$. The peptide sequence (RAD16-I, AcN-RADAR ADARADARADA-CONH ${ }_{2}$ ) promotes cell attachment ${ }^{16}$ and migration. ${ }^{17}$ It has been shown to support the differentiation of rat PC12 cells to form functional synapses analogous to rat primary hippocampal neurons, ${ }^{18}$ promote the development of cartilage from bovine chondrocytes in vitro, ${ }^{19}$ and induce the differentiation of rat liver progenitor cells into functional hepatocyte-like cells. ${ }^{20}$

In this paper, primary rat hepatocyte cultures on PuraMatrix were evaluated for their morphology, viability, and expression of hepatic functions. Two "gold standards" were used for comparison, namely, the collagen sandwich culture configuration and culture on Matrigel. Hepatocytes cultured on the PuraMatrix peptide hydrogel attached, migrated, and formed stable three-dimensional spheroids of $80 \mu \mathrm{m}$ of mean diameter. Results from this study showed that PuraMatrix promoted higher hepatic functions (albumin and urea) early in culture than the collagen sandwich cultures did. Subsequently, hepatic functions were similar to the collagen sandwich and Matrigel systems.

\section{MATERIALS AND METHODS}

\section{Materials}

Dulbecco's modified Eagle's medium (DMEM) containing $4.5 \mathrm{~g} / \mathrm{L}$ glucose, fetal bovine serum (FBS), penicillin, streptomycin, and trypsin-EDTA (ethylenediaminetetraacetic acid) was obtained from Invitrogen Life Technologies (Carlsbad, CA). Unless otherwise noted, all other chemicals were purchased from Sigma-Aldrich Chemicals (St. Louis, MO).

\section{Primary rat hepatocyte isolation}

Two- to 3-month-old Female Lewis rats (Charles River Laboratories, Wilmington, MA) weighing 180 to $200 \mathrm{~g}$ were used as a hepatocyte source and were maintained in accordance with the National Research Council guidelines. Experimental protocols were approved by the Subcommittee on Animal Care, Committee on Research, Massachusetts General Hospital. Using a modification of the two-step collagenase perfusion method, ${ }^{21,22}$ which involves purification of the cell suspension by means of centrifugation over Percoll, we routinely isolated approximately 200 million cells from one rat with viability between $85 \%$ and $98 \%$, as judged by trypan blue exclusion.

\section{Hepatocyte culture in collagen sandwich, Matrigel, and PuraMatrix}

Type I collagen was prepared by extracting acid-soluble collagen from Lewis rat-tail tendons. ${ }^{2}$ To create a thin layer of collagen gel in 12-well tissue culture plates, $200 \mu \mathrm{L}$ of an ice-cold mixture of one part of $10 \times$ concentrated DMEM and nine parts of $1.25 \mathrm{mg} / \mathrm{mL}$ rat-tail tendon type I collagen were evenly distributed over the bottom of each well. The plates were incubated at $37^{\circ} \mathrm{C}$ for $60 \mathrm{~min}$ to induce collagen gelation before cell seeding.

To form the nanostructured hydrogel, $600 \mu \mathrm{L}$ of $0.25 \%$ (v/v) PuraMatrix (a gift from 3DM, Cambridge, MA) was uniformly spread over each well of 12-well tissue culture plates. The medium was slowly added to each well by dripping along the wall to promote gelation according to the manufacturer's protocol. The medium was changed twice to equilibrate the growth environment to physiological $\mathrm{pH}$.

Two modified culture conditions were also examined to explore ways to control the size of hepatocyte spheroids and their effects on hepatic functions. The first method was to mix one part of $1.25 \mathrm{mg} / \mathrm{mL}$ type I collagen with nine parts of $0.25 \%$ PuraMatrix to generate a mixed gel. The same gelling method as for PuraMatrix was used to form the mixed gel. We also investigated the effect of dexamethasone (Dex) on hepatocyte morphology and function by adding $0.1 \mu \mathrm{M}$ Dex to the normal hepatocyte medium.

Matrigel (BD Biosciences, San Jose, CA) was also used as a control for primary hepatocyte culture. Matrigel ( $300 \mu \mathrm{L} /$ well) was uniformly spread over the bottom of an ice-cold 12 -well plate. The plate was transferred to a $37^{\circ} \mathrm{C}$ incubator for $30 \mathrm{~min}$ to allow gelation.

Each well of the 12 -well culture plates received $5 \times 10^{5}$ primary hepatocytes in suspension in $0.6 \mathrm{~mL}$ standard hepatocyte culture medium, which consisted of DMEM supplemented with $14 \mathrm{ng} / \mathrm{mL}$ glucagon, $7.5 \mu \mathrm{g} / \mathrm{mL}$ hydrocortisone, $0.5 \mathrm{U} / \mathrm{mL}$ insulin, $20 \mathrm{ng} / \mathrm{mL}$ epidermal growth factor, $200 \mathrm{U} / \mathrm{mL}$ penicillin, $200 \mu \mathrm{g} / \mathrm{mL}$ streptomycin, and $10 \%$ FBS. Cultures were incubated in $90 \%$ air/10\% carbon dioxide at $37^{\circ} \mathrm{C}$. For the double collagen gel culture configuration, a second layer of $250 \mu \mathrm{L}$ collagen was put on top of the cells the next day after seeding. Three wells of samples were maintained independently for each culture configuration for 3 weeks in each experiment. The medium was changed every $24 \mathrm{~h}$ and collected separately for each well every other day. 


\section{Hepatocyte viability and morphology by microscopy}

The morphology of hepatocytes cultured in the collagen sandwich and on the PuraMatrix hydrogel was visualized by phase and fluorescence microscopy on a Zeiss Axiovert 200 microscope following live/dead staining using calcein and ethidium homodimer-1 (Invitrogen-Molecular Probes, Carlsbad, CA). The hepatocyte spheroid size was measured from phase contrast images using an image analysis software, Metamorph (Universal Imaging, Downingtown, PA).

To study the detailed surface morphology of hepatocyte spheroids in PuraMatrix cultures, scanning electron microscopy was used. Primary hepatocytes $\left(2.5 \times 10^{5}\right)$ were cultured in a \#1.5 coverglass four-unit cell culture chamber (Nalge Nunc, Naperville, IL) using $300 \mu \mathrm{L} 0.25 \%$ PuraMatrix. Seven days after seeding, the cells in the chamber were fixed with $4 \%$ paraformaldehyde in phosphatebuffered saline (PBS) for $30 \mathrm{~min}$ and vacuum dried with DRIERITE desiccants (W.A. Hammond Drierite, Xenia, $\mathrm{OH})$ for $2 \mathrm{~h}$. Scanning electron microscope was then used to take the images.

\section{Hepatocyte functions}

Albumin concentration in the collected medium samples was analyzed using a competitive enzyme-linked immunosorbent assay. ${ }^{2}$ Albumin protein (cat. \#55952) and its antibody (cat. \#55776) were purchased from MP Biomedicals (Solon, OH). Urea concentration was determined via its specific reaction with diacetyl monoxime with a commercially available assay kit (Fisher Scientific, Pittsburgh, PA; cat. \#SB0580-250). The absorbance was measured with a Thermomax microplate reader (Molecular Devices, Sunnyvale, CA).

Cytochrome P450 (CYP450) function was evaluated based on the ethoxyresorufin $o$-deethylase (EROD) activity, which is chiefly catalyzed by the isoenzyme CYP450IA1. The activity was measured as the rate of resorufin formation from the ethoxyresorufin substrate (Invitrogen-Molecular Probes). Briefly, $2 \mu \mathrm{M}$ of the CYP450IA1 inducer 3methylcholanthrene was added to the cell culture medium $48 \mathrm{~h}$ before the assay. A phenol red-free formulation of hepatocyte culture medium was used to avoid interference when reading the fluorescence signals. The substrate solution $(600 \mu \mathrm{L})$ containing $8 \mu \mathrm{M}$ ethoxyresorufin and $90 \mu \mathrm{M}$ dicumarol in Earle's balanced salt solution was added to each well after washing with PBS. After 5, 15, 35, and 45 min of incubation, $25 \mu \mathrm{L}$ of substrate solution was taken from each culture well in duplicate and dispensed in two wells of a 96-well plate. Fluorescence intensity was measured with an fMax fluorescence plate reader (excitation wavelength, $530 \mathrm{~nm}$; emission wavelength, $580 \mathrm{~nm}$; Molecular Devices). Fluorescence intensity was converted to the resorufin concentration by comparison with a standard curve of resorufin fluorescence versus concentration ranging from 0 to $1000 \mathrm{nM}$. The slope $(\mathrm{nM} / \mathrm{min})$ of the fitted resorufin synthesis curve, corresponding to the rate of resorufin formation, was used as the metric of CYP450 activity.

\section{DPP IV immunostaining}

Primary hepatocytes were cultured on $0.25 \%$ PuraMatrix and in collagen sandwich using the same \#1.5 coverglass four-unit cell culture chamber as mentioned before. On day 7 after seeding, cells were fixed with $4 \%$ paraformaldehyde solution in PBS for $30 \mathrm{~min}$, washed thrice with PBS for $5 \mathrm{~min}$, and incubated with $0.1 \%$ Triton X-100 for $15 \mathrm{~min}$ to permeabilize the cell membrane, followed by three 5-min washes. Cells then were exposed to the blocking solution with $1 \%$ BSA and $3 \%$ donkey serum in PBS at $4^{\circ} \mathrm{C}$ overnight. The next day, the samples were washed thrice for $15 \mathrm{~min}$, and incubated with a mouse anti-rat dipeptidyl peptidase IV (DPP IV; Cell Sciences, Canton, MA) antibody diluted 1:10 in the blocking buffer at $4{ }^{\circ} \mathrm{C}$ overnight. Then, samples were washed thrice for $15 \mathrm{~min}$ followed by $4 \mathrm{~h}$ of incubation with a secondary fluorescein-conjugated donkey anti-mouse IgG antibody (Jackson ImmunoResearch Laboratories, West Grove, PA) diluted 1:100 in the blocking buffer at room temperature. After incubation, samples were washed thrice for $15 \mathrm{~min}$. The coverglass slide chamber was put directly on a Zeiss LSM Confocal microscope for imaging.

\section{JC-1 staining for mitochondrial membrane potential measurement}

JC-1 (5,5',6,6'-tetrachloro-1,1' $, 3,3^{\prime}$-tetraethylbenzimidazolylcarbocyanine iodide; Invitrogen-Molecular Probes) was used to assess mitochondrial membrane potential differences between monolayer cultures on collagen gel and spheroid cultures on PuraMatrix after hepatocyte isolation. JC-1, which exhibits green fluorescence in monomeric form, accumulates within polarized mitochondria, where it forms aggregates with red fluorescence. Mitochondrial membrane depolarization due to high permeability or decreased function can be observed as a decrease in the red/green fluorescence ratio. On the next day after $5 \times 10^{5}$ primary hepatocytes were seeded on a collagen gel or $0.25 \%$ PuraMatrix in a well of a 12-well plate, cells were stained with $300 \mu \mathrm{L}$ of $10 \mu \mathrm{g} / \mathrm{mL} \mathrm{JC}-1$ solution in the medium for $10 \mathrm{~min}$ in a $37^{\circ} \mathrm{C}$ incubator. The cells were then washed twice with warm PBS and imaged using the Zeiss Axiovert 200 microscope. Quantitative fluorescence image analysis of the green and red fluorescence channels was performed using Metamorph to obtain the ratio of red/green fluorescence intensity for each culture method.

\section{Statistics}

Values are expressed as mean \pm standard deviation (SD), and statistical testing was performed using $t$-test with $\alpha=0.05$ for significance. 


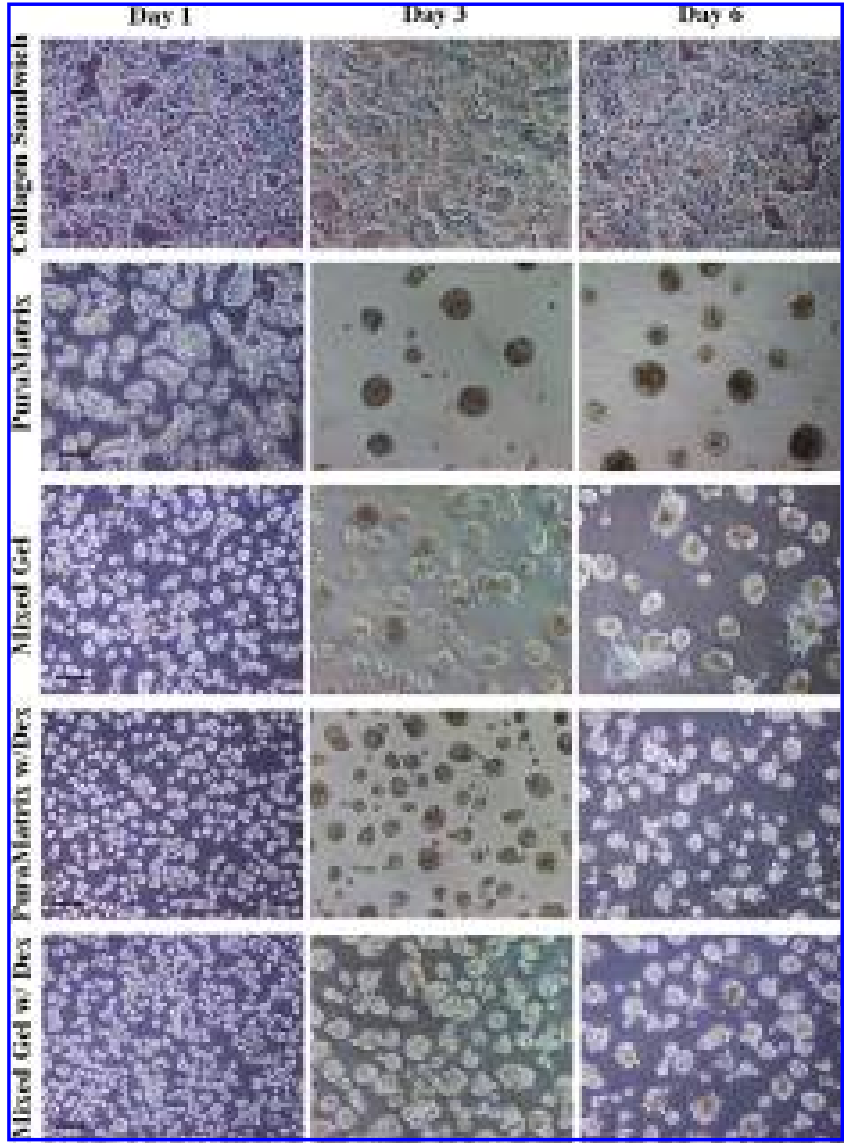

FIG. 1. Phase contrast images of primary hepatocytes cultured in the collagen sandwich, on $0.25 \%$ PuraMatrix gel, or on a mixed gel (one part of $1.25 \mathrm{mg} / \mathrm{mL}$ type I collagen mixed with nine parts of $0.25 \%$ PuraMatrix) in the hepatocyte medium without or with dexamethasone (Dex) on days 1, 3, and 6 after seeding. Scale bar is $100 \mu \mathrm{m}$. All images were captured at the same magnification. Color images available online at www.liebertpub.com/ten.

\section{RESULTS}

\section{Primary hepatocyte morphology in collagen sandwich and on PuraMatrix}

Hepatocyte morphology was captured by phase contrast and scanning electron microscopy. Figure 1 shows significant morphological differences between the collagen sandwich and PuraMatrix culture configurations. Cells formed a monolayer in the collagen sandwich cultures, while cells migrated and formed three-dimensional spheroids of different sizes on PuraMatrix within 3 days after seeding. Quantitative measurement of day-6 spheroids on PuraMatrix from the phase contrast images resulted in $79.8 \pm 20.9 \mu \mathrm{m}$ spheroids with $24 \%$ of the spheroids being larger than $100 \mu \mathrm{m}$ (Fig. 2).

To avoid the formation of large spheroids with diameters greater than $100 \mu \mathrm{m}$, two approaches were investigated. First, we made the PuraMatrix scaffold more cell adherent

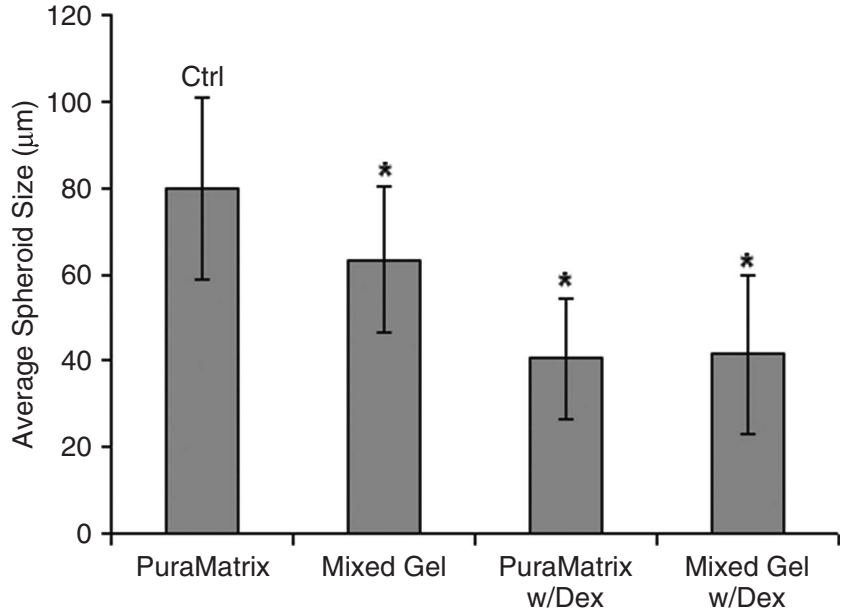

FIG. 2. Hepatocyte spheroid sizes on day 6 determined from phase contrast images in Figure 1. Asterisk (*) indicates a statistically significant difference $(p<0.05)$ compared to PuraMatrix cultures. The spheroid sizes are $79.8 \pm 20.9,63.4 \pm 16.9,40.5 \pm$ 14.3 , and $41.6 \pm 18.6 \mu \mathrm{m}$ (mean $\pm \mathrm{SD}$ ) for cells cultured on PuraMatrix $(n=17)$, mixed gel $(n=27)$, PuraMatrix with dexamethasone (Dex, $n=74$ ), and mixed gel with dexamethasone $(n=82)$, respectively.

by incorporating one part of $1.25 \mathrm{mg} / \mathrm{mL}$ type I collagen to nine parts of $0.25 \%$ PuraMatrix (labeled as Mixed Gel). Second, we used $0.1 \mu \mathrm{M}$ dexamethasone (Dex) to modulate the size of spheroids, as Dex was previously shown to reduce aggregate size in suspension cultures in spinner flasks. ${ }^{21}$ Figure 1 shows the morphology of the cultures on days 1, 3, and 6 after seeding, and Figure 2 presents the quantified results of spheroid size on day 6 of culture. The addition of both collagen and Dex reduced spheroid size, with average diameters of $63.4 \pm 16.9 \mu \mathrm{m}$ and $40.5 \pm 14.3 \mu \mathrm{m}$, respectively. Importantly, no spheroids $>100 \mu \mathrm{m}$ were observed in the modified culture conditions.

Detailed surface morphology of hepatocyte spheroids cultured on PuraMatrix, as visualized by scanning electron microscopy, is shown in Figure 3. Figure 3A shows bumpy surfaces of spheroids and the interaction between PuraMatrix and the spheroids. Several spheroids appeared to be halfway trapped inside the PuraMatrix gel, indicating that some of the hepatocytes migrated into the hydrogel after seeding, while others remained on the surface. Figure 3B, a higher magnification image, shows that individual hepatocytes are distinguishable on the surface of the spheroids, but not in the inner layer. Multiple micropores were observed on the surface of the spheroids (Fig. 3C), suggesting the presence of channels into the spheroids.

\section{Hepatocyte viability in spheroids on PuraMatrix}

Staining with calcein AM (fluorescent green) for live cells and ethidium homodimer-1 (fluorescent red) for dead cells 


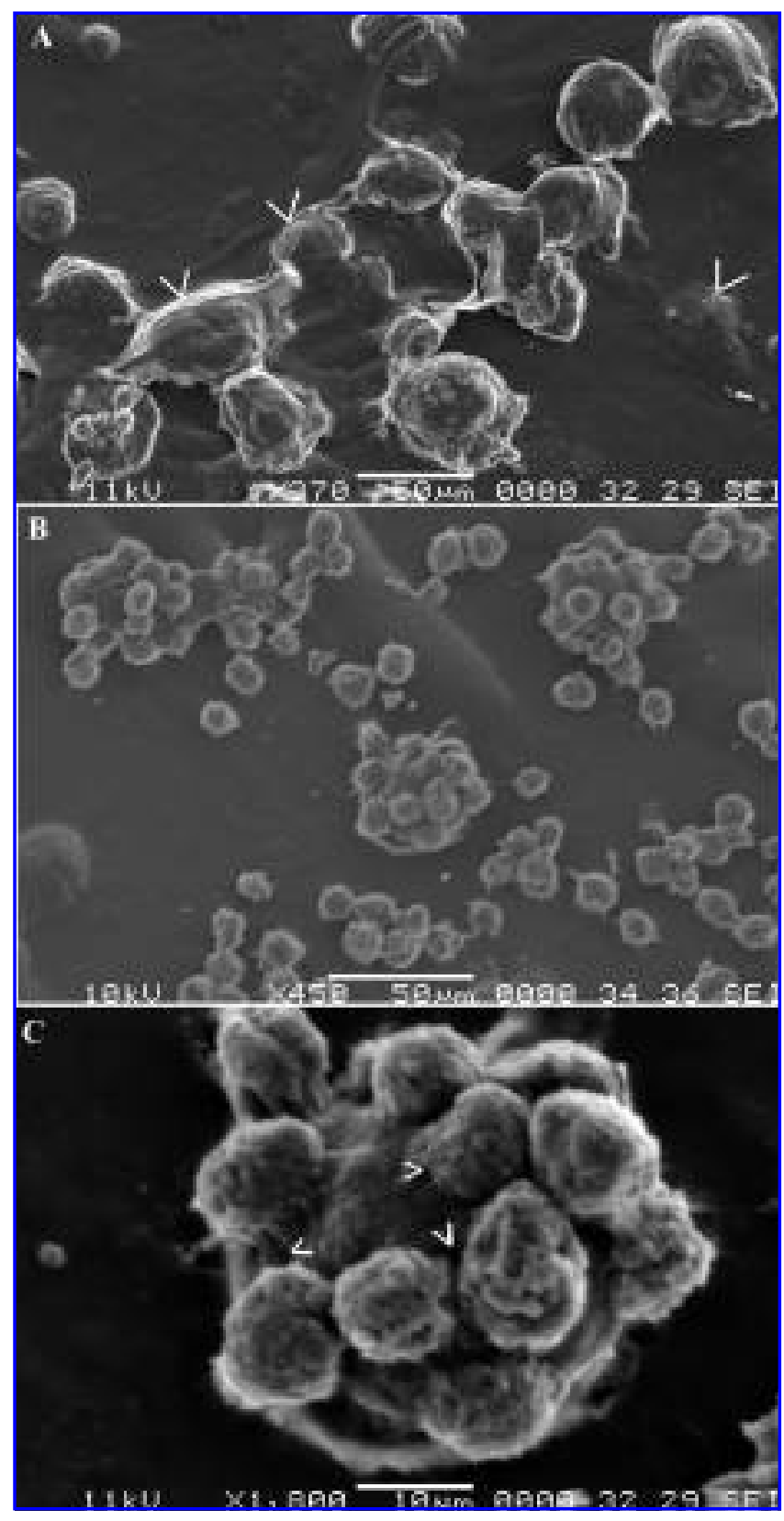

FIG. 3. Scanning electron microscopy images of hepatocyte spheroids formed on PuraMatrix. (A) Spheroids attached on the surface and, in some cases, trapped below the surface (white arrows) of the PuraMatrix; note the irregular surface. (B) Individual hepatocytes that provide the bumpy surface appearance. (C) Holes (white arrows) on the surface of a spheroid.

was used to assess cell viability. Fluorescence images of the stained hepatocyte spheroids cultured on PuraMatrix 1, 7, 11, and 18 days after seeding are shown in Figure 4. Most hepatocyte spheroids were bright green throughout the 3 weeks of culture, indicating high viability for that culture duration.
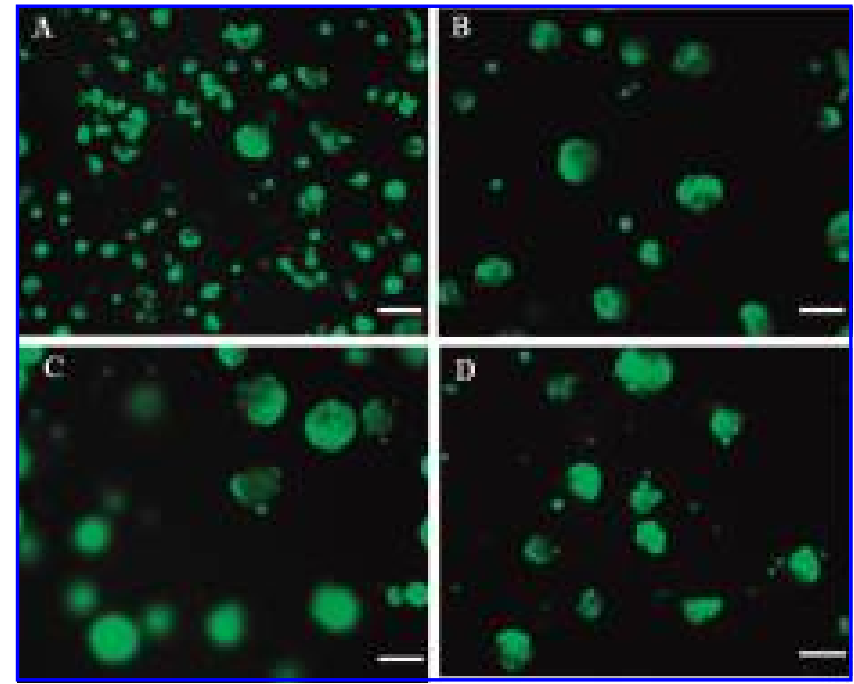

FIG. 4. Live/dead fluorescent staining of hepatocytes cultured on PuraMatrix hydrogel on $(\mathbf{A})$ day $1,(\mathbf{B})$ day $7,(\mathbf{C})$ day 11 , and $(\mathbf{D})$ day 18. Cells stained with calcein AM (green) are alive, while cells stained with ethidium homodimer-1 (red) are dead. Scale bar is $40 \mu \mathrm{m}$. Color images available online at www.liebertpub.com/ten.

\section{Hepatic function comparison among collagen sandwich, PuraMatrix, or Matrigel cultures}

Normalized albumin and urea secretion of primary hepatocytes cultured in the collagen sandwich and on $0.25 \%$ PuraMatrix as well as on Matrigel are shown in Figure 5. Experiments for hepatic function comparisons of PuraMatrix versus collagen sandwich cultures and PuraMatrix versus Matrigel cultures were performed using hepatocytes from different isolations. To compare the results from three culture configurations from different experiments, data in each experiment were normalized using the average albumin or urea value of the PuraMatrix cultures from day 8 to 21 in the same experiment as the base, since every experiment had the PuraMatrix culture in common and its functions reached the plateau on day 8 onward. In other words, the data shown in Figure 5 are average ratios of albumin or urea products to the base on different days in three culture configurations, PuraMatrix, collagen sandwich, and Matrigel.

Comparison between PuraMatrix and collagen sandwich cultures shows that cells cultured on PuraMatrix have higher albumin and urea production during the first week after seeding, suggesting faster functional recovery, than those cultured in collagen sandwich cultures. However, albumin and urea secretion values for both collagen sandwich and PuraMatrix cultures reach a similar plateau afterward. These experiments were independently repeated twice using hepatocytes from different isolations. With three independent samples in each experiment, the total sample number for the collagen sandwich culture is nine.

Albumin and urea production by primary hepatocytes cultured on Matrigel and $0.25 \%$ PuraMatrix is also compared 


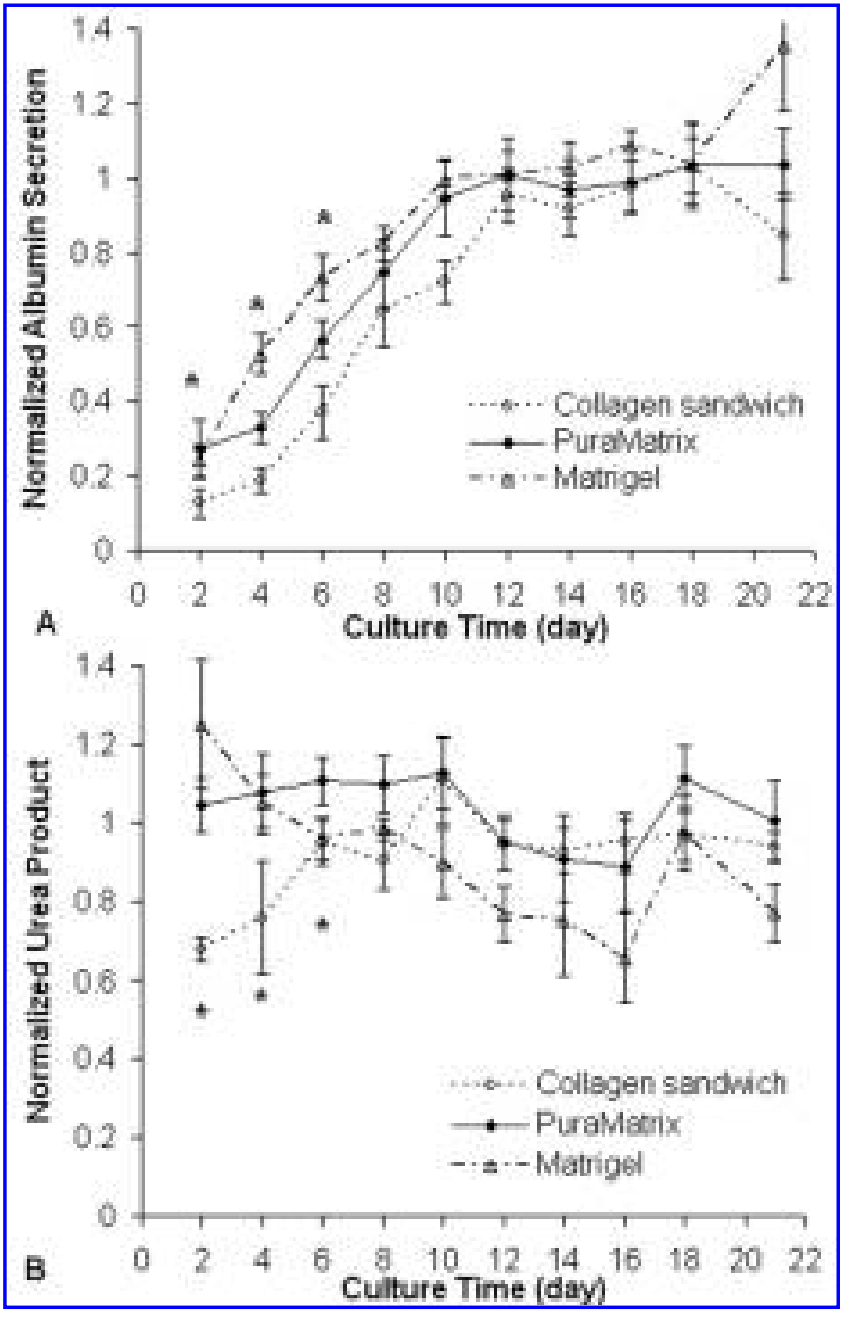

FIG. 5. Normalized albumin (A) and urea (B) production by primary hepatocytes cultured in the collagen sandwich, on $0.25 \%$ PuraMatrix, or on Matrigel. Data shown are mean \pm SD $(n=9$ for collagen sandwich, $n=15$ for PuraMatrix, and $n=6$ for Matrigel). Asterisk (*) indicates a statistically significant difference $(p<$ $0.05)$ between PuraMatrix and collagen sandwich cultures on the same day.

in Figure 5. Cells cultured on Matrigel showed slightly higher albumin production on days 4 and 6 after seeding, and then reached the plateau at the same level on day 8 and after. Urea function in PuraMatrix cultures was similar to Matrigel cultures up to 3 weeks after primary hepatocyte seeding. The same experiments were independently repeated using hepatocytes from different isolation. Therefore, the total sample number for the Matrigel culture is 6 and for the PuraMatrix culture is 15 .

\section{Hepatic function comparison for modified culture conditions}

Albumin and urea production up to 12 days after seeding is shown in Figure 6 for the modified culture conditions. Hepatocyte spheroids cultured on mixed gels had similar
A
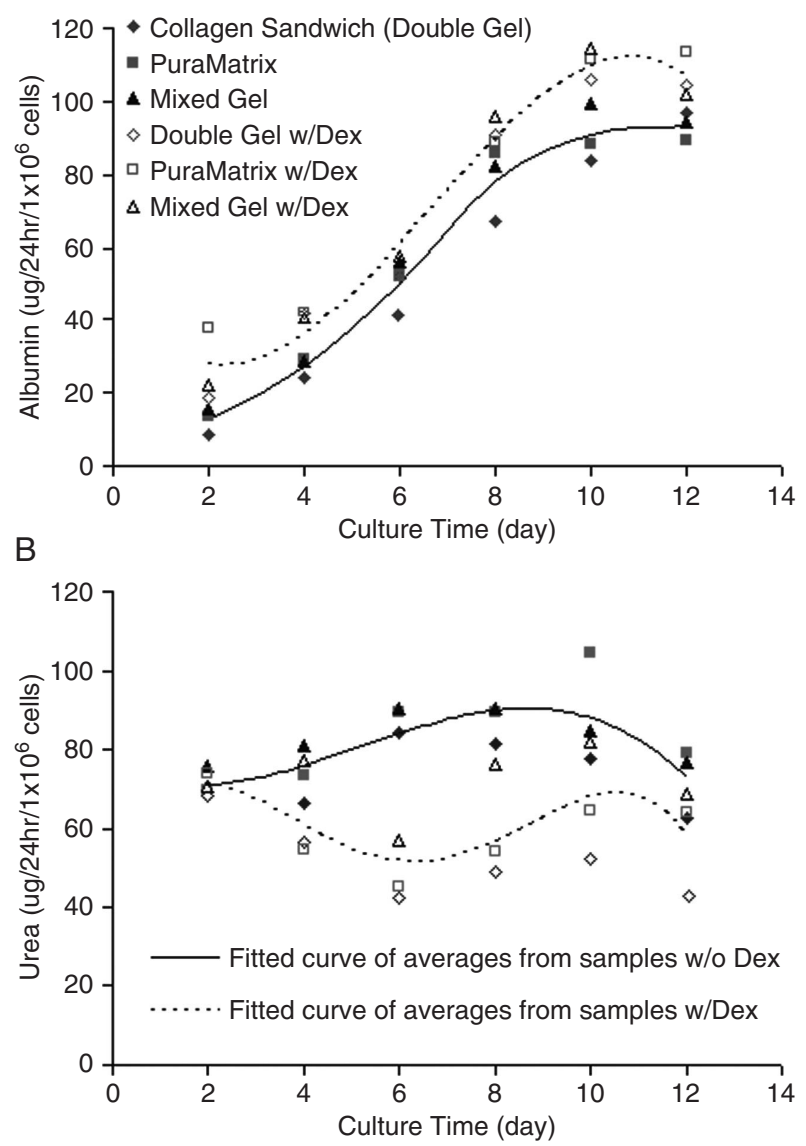

FIG. 6. Albumin (A) and urea (B) production by primary hepatocytes in the hepatocyte culture medium with or without $0.1 \mu \mathrm{M}$ Dex in the collagen sandwich, on $0.25 \%$ PuraMatrix, and on mixed gel (one part of $1.25 \mathrm{mg} / \mathrm{mL}$ type I collagen mixed with nine parts of $0.25 \%$ PuraMatrix). Each data point shown is the mean $(n=3)$ from a representative experiment. Solid lines are the fitted curves for the albumin/urea values of cultures in regular hepatocyte culture medium, while dashed lines are the fitted curves for the cultures in medium supplemented with $0.1 \mu \mathrm{M}$ Dex. Coefficients for the four fitted curves are $0.9862,0.9754,0.914$, and 0.8986 for the dashed and solid lines in (A), and solid and dashed lines in (B), correspondingly.

albumin and urea secretion rates compared with standard PuraMatrix cultures. The trend of lower initial function (up to day 8) by hepatocytes cultured in the collagen sandwich was also observed. In general, $0.1 \mu \mathrm{M}$ dexamethasone (Dex) moderately increased the albumin function in all culture configurations but suppressed the urea production. Hepatocyte spheroids on mixed gel and PuraMatrix resumed normal urea secretion levels on days 8 and 12, while levels of collagen-sandwiched hepatocytes remained low. The experiments were repeated once using hepatocytes from a different isolation, and the same trend was observed. The results of two independent experiments using hepatocytes from two different isolations could be lumped together by normalization with function values in the collagen sandwich culture as controls. However, absolute concentrations of 


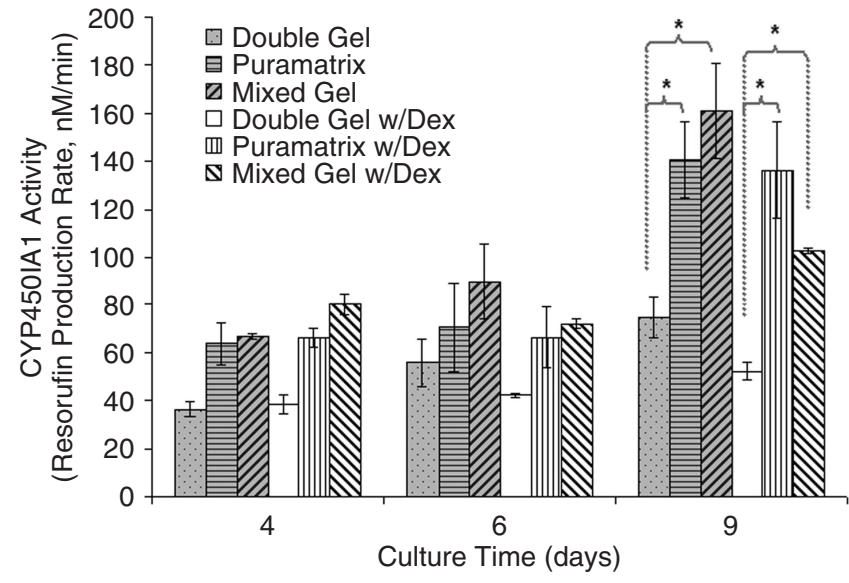

FIG. 7. Comparison of the induction of EROD activity by 3methylcholanthrene in hepatocytes cultured in the collagen sandwich, on PuraMatrix, or on mixed gel using the standard hepatocyte medium with or without $0.1 \mu \mathrm{M}$ Dex. Data shown are the mean $\pm \mathrm{SD}(n=4)$. Asterisk $(*)$ indicates a statistically significant difference $(p<0.01)$ compared to collagen sandwich cultures using the same medium on day 9 after seeding.

albumin and urea secretion would be lost after data normalization, which will make data comparison between different research labs very difficult.

Figure 7 shows that hepatocytes on PuraMatrix have a significantly higher $(p<0.01)$ CYP450IA1 activity than those on the collagen sandwich cultures on days 4,6 , and 9 regardless of whether the medium was supplemented with Dex or not. Clearly, the recovery of CYP450 activity in the collagen sandwich culture and on the mixed gel was inhibited by Dex. On the other hand, cultures on PuraMatrix did not show a Dex-mediated suppression of CYP450. The CYP450 activity experiments were independently repeated twice using hepatocytes from different isolations, and the same trend was also observed.

\section{Distribution of the apical membrane protein DPP IV}

The distribution of DPP IV, an apical membrane protein, was assessed by anti-DPP IV immunostaining of collagen sandwich and PuraMatrix cultures. Hepatocytes in both culture methods maintained membrane domain polarity (Fig. 8A, B). Interestingly, no DPP staining was observed at the cell-PuraMatrix contacts. In Figure 8B, there was no DPP IV staining at the edge of the spheroids, where hepatocytes interact with the PuraMatrix hydrogel.

\section{Mitochondrial membrane potential recovery after isolation on collagen gel and PuraMatrix}

Given the faster recovery of albumin and urea function by hepatocytes seeded on PuraMatrix than those seeded on the collagen sandwich, we hypothesized that the mitochondrial
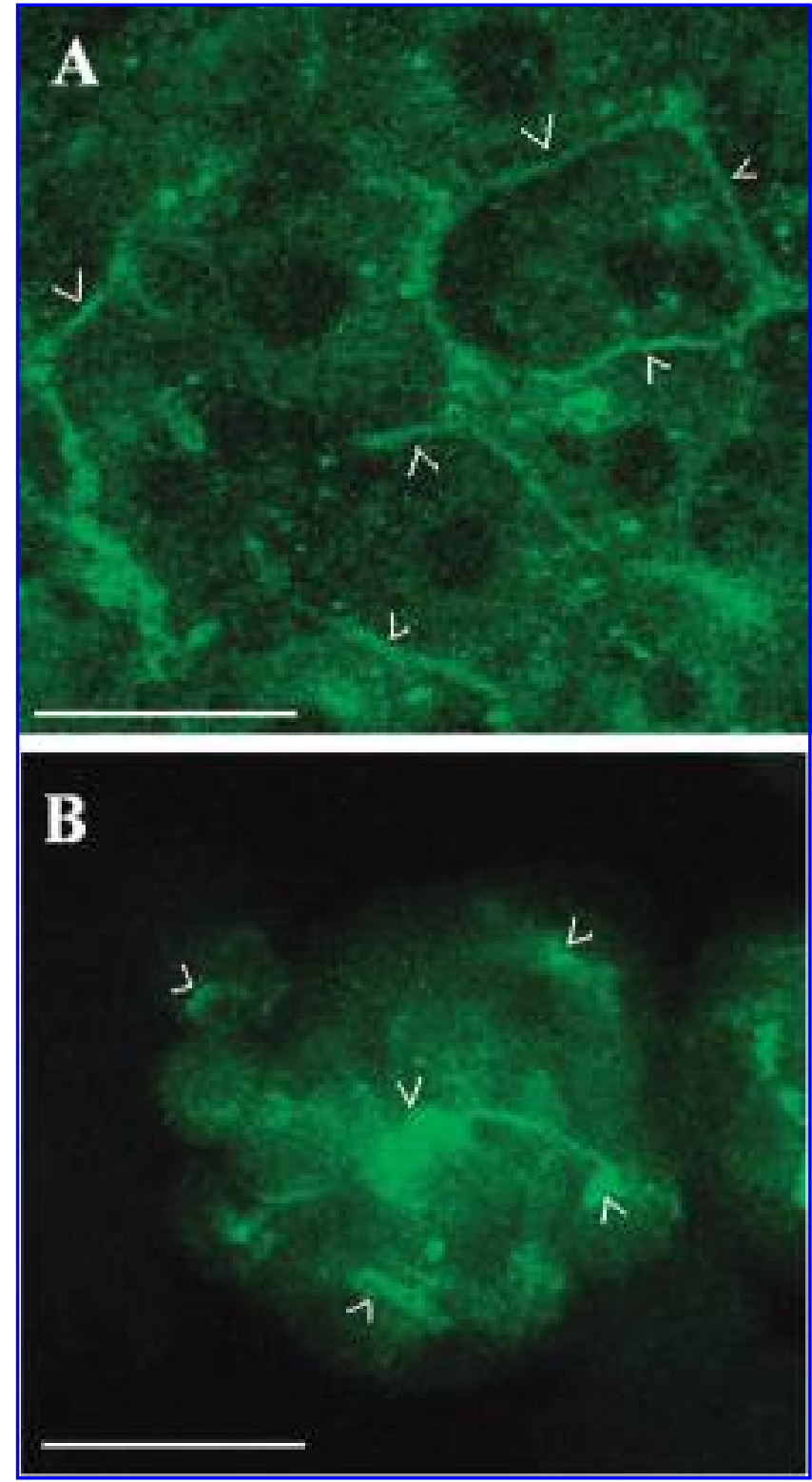

FIG. 8. Projected confocal images of hepatocytes immunostained for dipeptidyl peptidase IV (DPP IV) in (A) collagen sandwich cultures and (B) hepatocyte spheroids on PuraMatrix gels. Cultures were fixed on day 7 after seeding. Arrows point to areas where DPP IV is expressed. Scale bar is $25 \mu \mathrm{m}$. Color images available online at www.liebertpub.com/ten.

energy production may be better preserved in the PuraMatrix system. Thus, we performed JC-1 staining of hepatocyte cultures in these two systems to assess the recovery of the mitochondrial membrane potential 1 day after seeding. Figure 9 shows no difference in green fluorescence emission between the two systems, while the PuraMatrix cultures exhibited a much higher red fluorescence signal. Quantitative fluorescence image analysis shows a red/green fluorescence ratio approximately twice for the PuraMatrix cultures 


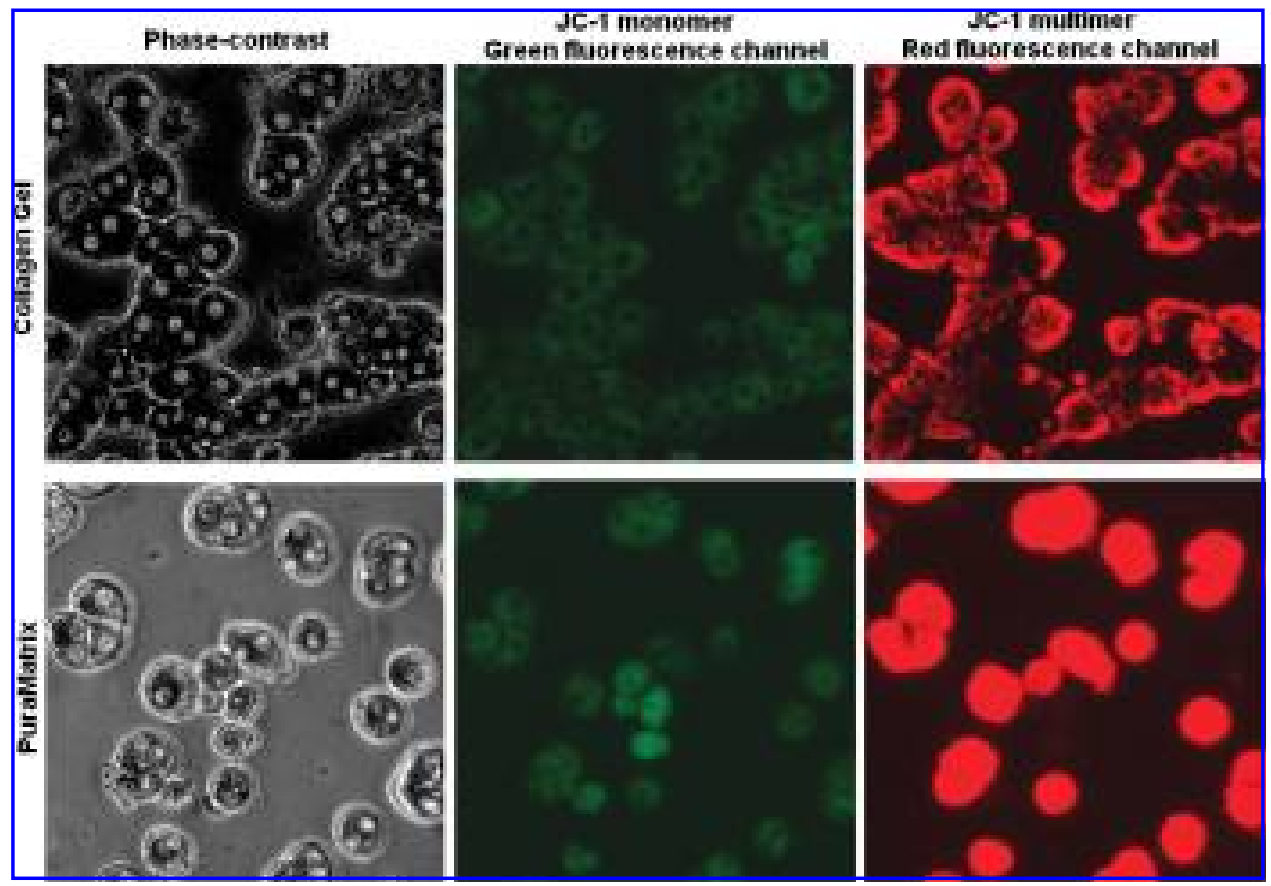

FIG. 9. JC-1 staining of primary hepatocytes cultured on a type I collagen gel and PuraMatrix 1 day after isolation and seeding shown by phase contrast images, green fluorescence images of the same field for JC-1 monomer distribution, and red fluorescence images for JC-1 multimer distribution.

compared to the collagen sandwich cultures (3.3 vs. 1.7 arbitrary units, respectively). These results suggest that the mitochondrial membrane potential of the hepatocytes cultured on PuraMatrix is higher than that of those cultured in the collagen sandwich early after seeding.

\section{DISCUSSION}

In this study, substrates made of the self-assembling peptide hydrogel PuraMatrix were evaluated as a biomaterial for the primary rat hepatocyte in vitro culture. Freshly isolated hepatocytes seeded on top of the hydrogel migrated and formed stable three-dimensional spheroids of $80 \mu \mathrm{m}$ average diameter within 3 days. The majority of hepatocytes remained viable, and apical polarity was maintained for more than 3 weeks of culture. The detailed surface morphology of hepatocyte spheroids on PuraMatrix revealed a bumpy surface with distinguishable individual hepatocytes and pores at cell-cell junctions. Similar pores have been observed on hepatocyte aggregates formed in spinning flasks, ${ }^{10}$ although the latter aggregates had a smooth surface. PuraMatrix spheroid cultures exhibited higher albumin secretion and urea production in the first week after seeding than collagen sandwich cultures did, which indicated a faster recovery from the isolation stress on PuraMatrix. In addition, the CYP450IA1 activity, a major detoxification pathway, was significantly higher on PuraMatrix than in the collagen sandwich during both the first and the second weeks of culture. We also compared the performance of PuraMatrix with that of Matrigel, which is often used to maintain hepatocyte function in vitro. Our results showed that albumin secretion was slightly higher on Matrigel than on PuraMatrix early after seeding, but from day 6 onward albumin and urea production was similar in both configurations. Given the well-defined and synthetic nature of PuraMatrix, these results suggest that PuraMatrix is a biomaterial with a good potential for a long-term hepatocyte culture in several applications, including clinical bioartificial liver (BAL) development and in vitro drug metabolism and toxicity evaluation.

The mechanism whereby spheroid culture on PuraMatrix helps hepatocyte recovery from the isolation injury, resulting in higher initial function, remains to be elucidated. The higher mitochondrial membrane potential 1 day after seeding suggests better preserved mitochondrial integrity in PuraMatrix cultures. Thus, the metabolic machinery in hepatocytes on PuraMatrix may be potentially able to generate more energy in the form of adenosine triphosphate (ATP), which could favorably impact on many repair processes, such as restoration of ion balance via ATP-dependent pumps.

PuraMatrix is a self-assembling peptide nanofiber ( $\sim 10 \mathrm{~nm}$ ) hydrogel scaffold consisting of over $99.75 \%$ water and 50-200 nm pores. ${ }^{15}$ The peptide sequence enables cell attachment and migration, thus enabling the rapid formation of cell-cell contacts. It has been reported that different cells cultured on PuraMatrix formed patterns similar to their in vivo morphology. For example, microvascular endothelial cells formed capillary-like networks, ${ }^{23}$ while 
mouse embryonic mesenchymal cells (3T3-J2) formed monolayers (unpublished observations). Our results show that primary rat hepatocytes tend to form small aggregates where individual hepatocytes express in vivo-like cell membrane polarity, which is a similar morphology as hepatocytes cultured on Matrigel.

The main limitation of the hepatocyte aggregate/spheroid as a large-scale culture system is oxygen transport. Prior studies suggest that the maximum diameter of a hepatocyte aggregate without an anoxic core is about $100 \mu \mathrm{m} .{ }^{24}$ Most existing methods for producing hepatocyte aggregates typically generate mean diameters ranging from 100 to $150 \mu \mathrm{m} .{ }^{1,4,10}$ Although PuraMatrix yielded an average diameter of $80 \mu \mathrm{m}$, about one quarter of the spheroids exceeded $100 \mu \mathrm{m}$. We found that the aggregate size could be reduced by about $35 \%$ by adding a small amount of type I collagen to the PuraMatrix. Collagen is a relatively stiff material whose fiber diameter is approximately $300 \mathrm{~nm}$, and is also extremely adherent to hepatocytes; as a result, it is likely that collagen incorporation into the PuraMatrix reduces hepatocyte migration, and results in smaller hepatocyte spheroids. The addition of $0.1 \mu \mathrm{M}$ dexamethasone (Dex) to the medium reduced the aggregate size by about $60 \%$. Although the underlying mechanism is unclear, this result is consistent with one report using suspension cultures in spinner flasks. ${ }^{25}$ This prior publication also reported a higher albumin secretion rate with Dex, which agrees with our data showing that albumin production was increased in the presence of Dex for all culture configurations. The albumin gene promoter region is known to have a glucocorticoid response element, which may mediate this observed increase. ${ }^{26}$ Another possible explanation may be due to the antiinflammatory properties of Dex, which may reverse albumin suppression induced by a proinflammatory or acute phase response triggered by the isolation procedure. ${ }^{27}$ On the contrary, we did not observe more urea production in the presence of Dex, unlike what was reported by Abu-Absi et al. ${ }^{25}$ Interestingly, Dex had no effect on the CYP450 activity in PuraMatrix cultures, but inhibited the recovery of CYP450 function beyond day 4 in the two culture configurations using type I collagen. Clearly, the effects of Dex on hepatic functions in hepatocyte in vitro culture remain to be elucidated; however, we propose that including $0.1 \mu \mathrm{M}$ Dex in the hepatocyte culture medium for the first 3 to 4 days after seeding is an efficient way to control the size of the spheroids on PuraMatrix and help cells recover from isolation injury.

Despite the advantages of using PuraMatrix to culture primary hepatocytes for BAL applications, we observed the detachment of spheroids from the PuraMatrix gel in cultures 3 weeks after seeding. Material (PuraMatrix) degradation seems to be a major cause for the detachment. Further, a major factor that limits PuraMatrix in such applications is its low mechanical strength due to its high water concentration $(>99 \%)$. Currently, we are developing cocultures of hepatocytes with nonparenchymal cells to further enhance the hepatic function on PuraMatrix. The cocultures may stabilize the material due to secretion of extracellular matrix from stellate and endothelial cells, and also increase hepatic functions due to growth factors secreted by these cells. Currently, we can maintain primary hepatocyte cocultures for $\sim 45$ days (data not shown).

In summary, this is the first detailed investigation of the use of a self-assembling peptide-based nanofiber scaffold hydrogel (PuraMatrix) for primary rat hepatocyte culture. Comparing the morphology and function of hepatocytes on PuraMatrix to those in the collagen sandwich and on Matrigel substrates showed that PuraMatrix enables rapid functional recovery of hepatocytes after isolation, and enables the formation of small hepatocyte aggregates that maintain a high level of stable liver-specific function. Given that PuraMatrix is a well-defined synthetic substrate, it represents a promising tool for engineering hepatocyte cultures for medically relevant applications, including toxicology and metabolic analyses of drugs, as well as hepatocyte-based therapies.

\section{ACKNOWLEDGMENTS}

We gratefully acknowledge the kind gift of PuraMatrix from the manufacturer 3DM (Cambridge, MA). This work was partially supported by the Shriners Hospitals for Children, and the National Institutes of Health grants R01DK43371 and P41EB002503.

\section{REFERENCES}

1. Allen, J.W., and Bhatia, S.N. Improving the next generation of bioartificial liver devices. Semin. Cell Dev. Biol. 13, 447454, 2002.

2. Dunn, J.C., Tompkins, R.G., and Yarmush, M.L. Long-term in vitro function of adult hepatocytes in a collagen sandwich configuration. Biotechnol. Prog. 7, 237-245, 1991.

3. Ryan, C.M., Carter, E.A., Jenkins, R.L., Sterling, L.M., Yarmush, M.L., Malt, R.A., and Tompkins, R.G. Isolation and long-term culture of human hepatocytes. Surgery 113, 48-54, 1993.

4. Landry, J., Bernier, D., Ouellet, C., Goyette, R., and Marceau, N. Spheroidal aggregate culture of rat liver cells: histotypic reorganization, biomatrix deposition, and maintenance of functional activities. J. Cell Biol. 101, 914-923, 1985.

5. Tong, J.Z., Sarrazin, S., Cassio, D., Gauthier, F., and Alvarez, F. Application of spheroid culture to human hepatocytes and maintenance of their differentiation. Biol. Cell 81, 77-81, 1994.

6. Glicklis, R., Shapiro, L., Agbaria, R., Merchuk, J.C., and Cohen, S. Hepatocyte behavior within three-dimensional porous alginate scaffolds. Biotechnol. Bioeng. 67, 344-353, 2000.

7. Moghe, P.V., Berthiaume, F., Ezzell, R.M., Toner, M., Tompkins, R.G., and Yarmush, M.L. Culture matrix configuration and composition in the maintenance of hepatocyte polarity and function. Biomaterials 17, 373-385, 1996. 
8. Coger, R., Toner, M., Moghe, P., Ezzell, R., and Yarmush, M. Hepatocyte aggregation and reorganization of EHS matrix gel. Tissue Eng. 3, 375-390, 1997.

9. Ammann, P., and Maier, P. Preservation and inducibility of xenobiotic metabolism in long-term cultures of adult rat liver cell aggregates. Toxicol. In Vitro 11, 43, 1997.

10. Wu, F.J., Friend, J.R., Hsiao, C.C., Zilliox, M.J., Ko, W.J., Cerra, F.B., and Hu, W.S. Efficient assembly of rat hepatocyte spheroids for tissue engineering applications. Biotechnol. Bioeng. 50, 404-415, 1996.

11. Berthiaume, F., Moghe, P.V., Toner, M., and Yarmush, M.L. Effect of extracellular matrix topology on cell structure, function, and physiological responsiveness: hepatocytes cultured in a sandwich configuration. FASEB J. 10, 1471-1484, 1996.

12. Roskelley, C.D., Desprez, P.Y., and Bissell, M.J. Extracellular matrix-dependent tissue-specific gene expression in mammary epithelial cells requires both physical and biochemical signal transduction. Proc. Natl. Acad. Sci. USA 91, 12378-12382, 1994.

13. Stoklosowa, S. Three dimensional tissue and organ models in vitro: their application in basic and practical research. Folia Histochem. Cytobiol. 39, 91-96, 2001.

14. Schindler, M., Nur, E.K.A., Ahmed, I., Kamal, J., Liu, H.Y., Amor, N., Ponery, A.S., Crockett, D.P., Grafe, T.H., Chung, H.Y., Weik, T., Jones, E., and Meiners, S. Living in three dimensions: 3D nanostructured environments for cell culture and regenerative medicine. Cell Biochem. Biophys. 45, 215227, 2006.

15. Zhang, S., Holmes, T., Lockshin, C., and Rich, A. Spontaneous assembly of a self-complementary oligopeptide to form a stable macroscopic membrane. Proc. Natl. Acad. Sci. USA 90, 3334-3338, 1993.

16. Zhang, S., Holmes, T.C., DiPersio, C.M., Hynes, R.O., Su, X., and Rich, A. Self-complementary oligopeptide matrices support mammalian cell attachment. Biomaterials 16, 1385-1393, 1995.

17. Semino, C.E., Kasahara, J., Hayashi, Y., and Zhang, S. Entrapment of migrating hippocampal neural cells in threedimensional peptide nanofiber scaffold. Tissue Eng. 10, 643-655, 2004.

18. Holmes, T.C., de Lacalle, S., Su, X., Liu, G., Rich, A., and Zhang, S. Extensive neurite outgrowth and active synapse formation on self-assembling peptide scaffolds. Proc. Natl. Acad. Sci. USA 97, 6728-6733, 2000.
19. Kisiday, J., Jin, M., Kurz, B., Hung, H., Semino, C., Zhang, S., and Grodzinsky, A.J. Self-assembling peptide hydrogel fosters chondrocyte extracellular matrix production and cell division: implications for cartilage tissue repair. Proc. Natl. Acad. Sci. USA 99, 9996-10001, 2002.

20. Semino, C.E., Merok, J.R., Crane, G.G., Panagiotakos, G., and Zhang, S. Functional differentiation of hepatocyte-like spheroid structures from putative liver progenitor cells in threedimensional peptide scaffolds. Differentiation 71, 262-270, 2003.

21. Seglen, P.O. Preparation of isolated rat liver cells. Methods Cell Biol. 13, 29-83, 1976.

22. Dunn, J.C., Yarmush, M.L., Koebe, H.G., and Tompkins, R.G. Hepatocyte function and extracellular matrix geometry: longterm culture in a sandwich configuration. FASEB J. 3, 174177, 1989.

23. Narmoneva, D.A., Oni, O., Sieminski, A.L., Zhang, S., Gertler, J.P., Kamm, R.D., and Lee, R.T. Self-assembling short oligopeptides and the promotion of angiogenesis. Biomaterials 26, 4837-4846, 2005.

24. Catapano, G. Mass transfer limitations to the performance of membrane bioartificial liver support devices. Int. J. Artif. Organs 19, 18-35, 1996.

25. Abu-Absi, S.F., Hu, W.S., and Hansen, L.K. Dexamethasone effects on rat hepatocyte spheroid formation and function. Tissue Eng. 11, 415-426, 2005.

26. Nawa, K., Nakamura, T., Kumatori, A., Noda, C., and Ichihara, A. Glucocorticoid-dependent expression of the albumin gene in adult rat hepatocytes. J. Biol. Chem. 261, 16883-16888, 1986.

27. Paine, A.J., and Andreakos, E. Activation of signalling pathways during hepatocyte isolation: relevance to toxicology in vitro. Toxicol. In Vitro 18, 187-193, 2004.

Address reprint requests to: Martin L. Yarmush, M.D., Ph.D. Massachusetts General Hospital 55 Fruit St.

GRB 1401

Boston, MA 02114

E-mail: ireis@sbi.org 